Revista de Psicología y Educación / Journal of Psychology and Education, 2020, 15(1), 98-107 (www.rpye.es) Doi: https://doi.org/10.23923/rpye2020.01.189

ISSN: $1699-9517$

\title{
La Escala de Procrastinación Académica (EPA): validez y confiabilidad en una muestra de estudiantes Peruanos
}

\author{
Kimberly Trujillo-Chumán y Martín Noé-Grijalva* \\ Universidad César Vallejo-Perú
}

\begin{abstract}
Resumen: El estudio tuvo como objetivo evaluar las evidencias de validez y confiabilidad de la Escala de Procrastinación Académica en estudiantes Peruanos. El muestreo fue estratificado: 366 estudiantes de secundaria del distrito de Chimbote (Perú), de entre 12 y 17 años. Se halló que en la estructura factorial verifico un ajuste aceptable de un modelo final re-especificado (M8-1) con valores con mejor ajuste absoluto $\left(\mathrm{X}^{2} / \mathrm{gl}<5\right.$; $\mathrm{GFI}>, 95$; RMSEA $<, 08$ y SRMR $\left.<, 08\right)$, un ajuste comparativo más favorable (CFI y $T L I>$,95), además un ajuste parsimonioso con valores inferiores al primer modelo ( $A I C=201,13$ y 68,98). En su fiabilidad, se presenta los valores de consistencia interna según el coeficiente de consistencia interna Omega de .80, donde la valoración es aceptable. Se concluye que la Escala de Procrastinación Académica (EPA) es una medida que puede emplearse en la actividad de la Psicología educativa e investigativa, obteniendo una adecuada validez y puntuaciones fiables en población peruana.

Palabras clave: Procrastinación académica, validez, confiabilidad, cuestionario, Secundaria.
\end{abstract}

\section{The academic procrastination scale: validity and reliability in a sample of Peruvian students}

Abstract: The study aimed to evaluate the evidence of validity and reliability of the Academic Procrastination Scale in Peruvian students. The sampling was stratified: 366 high school students from the Chimbote district (Peru), between 12 and 17 years old. It was found that in the factorial structure I verify anacceptable adjustment of a re-specified final model (M8-1) with values with better absolute adjustment $\left(X^{2} / \mathrm{gl}<5\right.$; GFI > 95; RMSEA <, 08 and SRMR<,08), a more favorable comparative adjustment (CFI and TLI > 95), in addition to a parsimonious adjustment with values lower than the first model (AIC = 201.13 and 68.98). In its reliability, the internal consistency values are presented according to the Omega internal consistency coefficient of .80, where the valuation is acceptable. It is concluded that the Academic Procrastination Scale (EPA) is a measure that can be used in the activity of educational and research Psychology, obtaining adequate validity and reliable scores in the Peruvian population.

Keywords: Academic procrastination, validity, reliability, questionnaire, Secondary.

El escenario del colegio implica retos, cambios y desarrollo de competencias (Chan, $2011)$ que involucra niños y adolescentes que exponen la idea de realizar las cosas con mayor rapidez y en menor tiempo posible y que no requieran de mucho esfuerzo. Por esta razón, cuando exista la obligatoriedad de cumplir con alguna tarea, el estudiante

\footnotetext{
Recibido: 03/09/2019 - Aceptado: 13/11/2019 - Avance online: 19/12/2019

*Correspondencia: Martín Noé-Grijalva.

Universidad César Vallejo-Perú.

C.P: 7124, Trujillo, Perú.

E-mail: hnoe@ucv.edu.pe
}

Trujillo-Chumán, K. y Noé-Grijalva, M. (Avance Online). La escala de Procrastinación académica-EPA: validez y confiabilidad en una muestra de estudiantes Peruanos. Revista de Psicología y Educación, 15(1), 98-107, https://doi.org/10.23923/rpye2020.01.189 podría elegir por dos opciones: empezar la actividad y no concluirla, o no iniciar la tarea. El fenómeno descrito previamente es lo que se conoce como la procrastinación, a la actividad de postergar la tarea académica (Guevara, 2017).

Según Anders (2016, citado por Duran y Moreta, 2017) la palabra procrastinación viene del latín procrastinare, que significa "posponer o dejar ciertas actividades para otra oportunidad o instante". Steel \& Ferrari (2013) manifiestan que este fenómeno se muestra como una dificultad en los procesos 
de autorregulación "que provoca la demora voluntaria de actividades planificadas, aunque se anticipe una situación peor como consecuencia de la demora". Afecta a los alumnos en el plano escolar, sea por bajas calificaciones o ausentismo académico. A ello, Cardona (2015, p.26) afirma que "la procrastinaciòn representa una dificultad de autorregulación particularmente significativa, ya que describe la discrepancia entre las intenciones o planes de acción y las conductas que efectivamente lo llevarían a la práctica".

Si bien es cierto el problema de procrastinación se ha presentado a lo largo de los años, fue considerado por mucho tiempo como parte usual de la vida del ser humano. Sin embargo, con el surgimiento de la revolución industrial y el apogeo de la competitividad, se evidenció que afectaba notablemente en el desarrollo de las empresas (Ferrari \& Emmons, 1995). Es así, Garzón y Gil (2017) indican que una de las diferentes variables por las que los alumnos desistían de acudir a un centro de estudios, era producto de lo que actualmente llamamos procrastinación académica.

Estudios en países no occidentales como Turquía muestran que un $83 \%$ de estudiantes del nivel secundario procrastinan (Klassen \& Kuzucu, 2009). Onwuegbuzie (2004) menciona que el $41.7 \%$ de los alumnos que van a la Universidad procrastina sobre la forma en que escriben un trabajo final, el $39.3 \%$ sobre el estudio ante los exámenes y un $60 \%$ lo hace cuando intenta tener un ritmo semanal de lectura.

El fenómeno descrito también se manifiesta en nuestra población latina. En así, que Quant \& Sánchez (2012) indican que en nuestro continente aproximadamente el $61 \%$ de las personas procrastinan y el $20 \%$ lo hacen de manera crónica, siendo esto un patrón concurrente de comportamiento.

Ante ello podemos afirmar que la procrastinación es la tendencia en dejar las actividades académicas para después ir postergando la realización de la ejecución de la tarea generando ansiedad por el incumplimiento (Busko, 1998).
Por lo tanto, la procrastinación académica puede tener diversos factores motivacionales según Steel (2007). El factor de Expectativa alude a la forma en la que se visualiza la tarea a realizar; el de Valoración alude a que ciertas actividades académicas resultan tener mayor placer y atraer más la atención que otras, de esta manera el sujeto asigna un valor determinado a cada tarea a realizar; el de Impulsividad alude directamente a la motivación que tiene la persona hacia la tarea asignada, en consecuencia, de ello, se tiene una alta probabilidad de procrastinar, esto bajo la perspectiva que el trabajo tendrá como resulta una baja recompensa; el de Demora de la satisfacción es paralelo al tiempo de respuesta y los beneficios que pueda traer consigo el llegar a concluir una actividad. En cambio, Steel (2007) establece dos factores ambientales predecibles: Tiempo de recompensas y castigos; y Aversión a la tarea.

Existen diferentes modelos, que explican la visión de la procrastinación desde diferentes perspectivas y corrientes dentro de la Psicología (Skinner, 1977, citado por Carranza y Ramírez, 2013): en el modelo conductual, las conductas de postergación propiamente han sido retroalimentadas por diversos factores del ambiente, que han permitido la continuación de este tipo de acciones; en el modelo cognitivo de Wolters, la procrastinación implica un procesamiento de información disfuncional que involucra esquemas desadaptativos relacionados con la incapacidad y el miedo llevando al comportamiento de aplazamiento; en el modelo cognitiva-conductual (Ellis y Knaus, 1977; citado por Álvarez, 2010) los sujetos tienden a plantearse metas $u$ objetivos que son altas o poco reales que por consiguiente llevan al fracaso.

Según Schouwenburg (2004), hay dos tipos de procrastinación académica: esporádica y crónica. La primera se relaciona con actividades académicas concretas, debido a carencias en la gestión del tiempo. La segunda se refiere al hábito generalizado de demorar la dedicación al estudio. 
Luego la procrastinación académica presenta diferentes componentes, cognitivos, conductuales y afectivo-emocionales (Alegre, 2013). Estos tipos son descritos por Hernández (2016): el Componente cognitivo se refiere al uso continuo de racionalizaciones o excusas para postergar las acciones; el Componente conductual se vincula con la impulsividad, distracción y una notable incoherencia entre lo que se pretende hacer y lo que se terminar por ejecutar; el Componente emocional, tienden a fracasar en su actividad académica.

Por su parte, Ellis \& Knaus (2002) presentan una serie de características que permite identificar a las personas que procrastinan sus actividades académicas: Creencias irracionales, que conlleva a lo sujetos a que observen como personas incapaces de llevar a cabo una tarea; Ansiedad y catastrofismo, que se refiere a que el sujeto se siente cargado y cansado por las pocas probabilidades que halle para obtener algún éxito que le produzca satisfacción; Perfeccionismo y miedo al fracaso, cuando se posterga una actividad y esto se justifica por falta de tiempo por otro lado son perfeccionistas y autoexigentes que se trazan metas poco realistas; Rabia e Impaciencia, que son aquellas personas perfeccionistas, que al no poder cumplir con propósitos que se han fijado se muestran agresivos contra sí mismos; Necesidad de Sentirse Querido, que es la necesidad que sienten las personas de obtener logros, alcanzar metas, culminar propósitos con el fin de conseguir algún reconocimiento o aceptación de los demás; Sentirse Saturado, cuando llega el momento de entregar las tareas y hay una acumulación de trabajos que no se han finalizado.

La evidencia científica muestra que la procrastinación académica es vista por muchos como uno de los causantes del fracaso escolar, la deserción en la escuela, y de otros fenómenos que afectan la vida académica de los jóvenes a largo plazo (Rodríguez y Clariana, 2017).

Ante el problema expuesto, muchos investigadores quisieron brindar información e instrumentos que guiados por una teoría con mayor incidencia en el plano cognitivo conductual, pudieran apoyar en la correcta identificación de los diferentes factores que afectan a la población peruana. Es así, que se hizo una revisión de investigaciones psicométricas del procrastinación. Quinde (2014) realizo un estudio sobre propiedades psicométricas de la Escala de Procrastinación Académica, que encontró una adecuada capacidad de discriminación $(0,25-0,88)$ con una varianza total de $67,97 \%$, y un alfa a la escala total de 0,92). Guevara (2017) realiza un análisis factorial confirmatorio de la Escala de Procrastinación Académica y obtiene valores de ajuste insuficientes $(\mathrm{CFI}=.853$ y $\mathrm{GFI}=$ .922) para confirmar la medición de la variable por estructura bifactorial, y su confiabilidad alcanza valoraciones de muy respetable. Domínguez, Villegas y Centeno (2014), en su análisis factorial confirmatorio de la Escala de Procrastinación Académica, los resultados se ajustan a la estructura de dos dimensiones y la confiabilidad presenta valores elevados. Obtuvo una fiabilidad de 0.816 en el total; de ,821 en el primer factor de autorregulación académica; y de ,752 en el segundo factor de postergación de actividades.

Para la presente investigación, se eligió la Escala de Procrastinación Académica creada por Busko (1998), pero adaptada por Álvarez (2010), que reporta bondades psicométricas en una muestra de universitarios.

Considerando la existencia de estudios psicométricos que han trabajado la procrastinación académica en una población universitaria; el estudio psicométrico busca otro escenario en edad y contexto escolar. Además de verificar la fiabilidad y la validez de la estructura unifactorial de la Escala de Procrastinación Académica (Busko, 1998) adaptada por Álvarez (2010) que garanticen su uso en el contexto de educación secundaria y en población de adolescentes.

Los resultados permitirán aportar sobre las evidencia psicométricas de una escala en un contexto de educación básica, que permita la evaluación de la procrastinación académica. Además el instrumento servirá de ayuda al trabajo de la Psicología Educativa.

\section{MÉTODO}

El tipo de investigación es instrumental, que pretende o propone la adaptación de un instrumento o herramienta ya existente a 
un nuevo contexto de aplicación pertinente al momento actual. De modo que, con el suficiente sustento teórico y razones para investigar, se proponga una escala en beneficio general (Ato, López-García, \& Benavente, 2013).

\section{PARTICIPANTES}

Se contó con la participación de 366 adolescentes, estudiantes de Secundaria, siendo el muestreo de tipo intencional, de entre los 12 y los 19 años $(M=14,4 ; D T=1,50)$. El $50.2 \%$ eran mujeres y el $49.7 \%$ hombres. Un análisis de comparación de medias evidenció que no existe diferencias significativas en el promedio de las puntuaciones de procrastinación entre hombres y mujeres, $t_{(364)}=1,518 ; p=0,130 ; d=0,160 ;$ IC $95 \%$ $-0,347-2,694$. Los estudiantes pertenecen a 19 instituciones educativas de ChimbotePerú.

En relación a los temas éticos, se entregó un consentimiento informado previo al llenado del cuestionario en donde se le explicaba las condiciones del anonimato, y su participación voluntaria y el objetivo de la investigación, así como el uso de los resultados.

\section{INSTRUMENTO}

El instrumento que se utilizó en el trabajo de investigación es la Escala de Procrastinación Académica, creada por Busko (1998). Está constituida por 16 reactivos, con cinco alternativas de respuesta (nunca, pocas veces, a veces, casi siempre y siempre). Se puntúan mediante una escala de tipo Likert de cinco puntos. Presenta una fiabilidad a través del coeficiente Alfa de Cronbach de ,86. Su adaptación al español por Álvarez (2010), con una fiabilidad a través del coeficiente Alfa de Cronbach de ,80. La administración de la escala es de forma individual o colectiva. Generalmente, la realización del cuestionario lleva entre 8 y 12 minutos, y en el caso del tiempo total no supera los 17 minutos. Esto permite que se evalúe con fiabilidad la propensión hacia la procrastinación académica.

\section{PROCEDIMIENTO}

Luego de que el instrumento fue aplicado en las diferentes instituciones educativas de la ciudad de Chimbote (Perú), se realizó en primera instancia la eliminación de los test que no fueron llenados completamente. A los tests válidos se les asignó un número, para luego ingresar los datos en una hoja de cálculo del programa Excel. Luego se exportaron dichos datos al programa SPSS 24, para determinar la validez a través del análisis de la estructura interna, así como la media, desviación estándar y otras medidas. Además, como segundo paso se usó el programa AMOS 26 para conocer la asimetría y curtosis, y realizar el análisis factorial confirmatorio, con el objeto de determinar si el modelo propuesto por la autora Young se ajusta a la información recogida en la población estudiada.

Finalmente, la confiabilidad fue analizada mediante el coeficiente Omega, usando el programa $R$ versión 3.6.1. Las cargas factoriales fueron diferentes y las asimetrías estaban entre -1.5 a 1-5, cumpliéndose los requisitos para aplicar Omega.

\section{ANÁLISIS DE DATOS}

Siendo los ítems con respuesta en escala tipo Likert (variables ordinales), entonces se procedió aplicar el Análisis Factorial Confirmatorio con ayuda del método de Mínimos Cuadrados Generalizados, usando para ello la matriz de varianzas y covarianzas (Hair, Anderson, Tatham, y Black, 2010). Para la evaluación de los modelos, se utilizó como criterio las medidas de bondad de ajuste GFI $\geq$,95, AGFI $\geq$,95, RMSEA $\leq, 08, \mathrm{CFI} \geq$,95, TLI $\geq$,95 (Ruiz, Pardo y San Martin, 2010), SRMR $\leq, 08$ (Hu y Bentler, 1998) y $\chi^{2} / g$ l cuyos valores oscilan entre 2 (Tabachnick y Fidell, 2007) y 5 (Wheaton, Muthen, Alwin, \&Summers, 1977). Todo ello se calculó con ayuda del software estadístico AMOS 26. Además, se calculó a través del software estadístico $R$ versión 3.6.1 el coeficiente de fiabilidad Omega (McDonald, 2013) para estimar de forma puntual e interválica, al 95\% de confianza, la consistencia interna del instrumento, considerando valores 
mayores a .70 como aceptables (Campo-Arias y Oviedo, 2008).

\section{RESULTADOS}

\section{ANÁLISIS DESCRIPTIVO}

En la Tabla 1 se presentan los valores descriptivos de las puntuaciones derivadas de la aplicación de la Escala de Procrastinación Académica en estudiantes de Secundaria de Chimbote. La puntuación promedio inferior fue para el ítem 6 $(M=1.54)$ y la superior para el ítem $3(M=3.19)$. La desviación estándar oscila entre .99 y 1.31. Los valores de asimetría y curtosis se ubican dentro del intervalo +/- 2, a excepción del reactivo 6 que presenta un valor de curtosis ligeramente superior al intervalo mencionado $\left(g^{2}=3.02\right)$. Se estima que hay presencia de normalidad univarida. Finalmente, en lo que concierne a los valores de correlación ítem-factor, se presentan valores que varían de .22 a .58 (aceptables), a excepción de los reactivos $4,6,15$ y 16 que presentan valores inferiores a .20 (inaceptables) (Kline, 1998). Además, por medio del test de Mardia se comprobó que había normalidad multivariada (Korkmaz, Goksuluk y Zararsiz, 2015).

\section{ANÁLISIS FACTORIAL CONFIRMATORIO}

La escala que mide la variable procrastinación académica usada para la presente investigación está estructurada de 16 ítems agrupados en una sola dimensión, mismo que se suministró en una muestra de 366 participantes, del tal manera que de los puntajes obtenidos se realizó un análisis factorial confirmatorio por medio del método de Mínimos cuadrados Generalizados (Fernández, 2015; Lara, 2014). Se reporta el primer ajuste absoluto, por medio del el valor de chi cuadrado sobre grados libertad $\left(X^{2} / g l=5,79\right)$, el índice de bondad de ajuste $(\mathrm{GFI}=, 85)$; el error cuadrático medio de aproximación (RMSEA $=, 115)$, y el residuo estandarizado cuadrático medio $(S R M R=.093)$; el ajuste comparativo por medio de su índice $(C F I=.61)$ y el índice de Tuker-Lewis (TLI=.55).

Se procedió a presentar modelos reespecificados, debido a la presencia de pesos factoriales estandarizados bajos y errores correlacionados altos (Escobedo, Hernández, Estebané y Martínez, 2016). En el primer modelo re-especificado se retiraron los

\section{Tabla 1
Media, desviación estándar, asimetría, curtosis y correlación ítem-factor $(n=366)$}

\begin{tabular}{|c|c|c|c|c|c|}
\hline \multirow[t]{2}{*}{ Ítems } & \multirow[t]{2}{*}{ Media } & \multirow[t]{2}{*}{ DE } & \multirow[t]{2}{*}{ Asi } & \multirow[t]{2}{*}{ Curt } & $\begin{array}{l}\text { Ítem } \\
\text { Factor }\end{array}$ \\
\hline & & & & & $r_{\text {itc }}$ \\
\hline 1. Cuando tengo que hacer una tarea, normalmente la dejo para el último minuto & 2,81 & 1,08 &, 07 &,- 45 & 26 \\
\hline 2. Generalmente me preparo por adelantado para los exámenes. & 2,91 & 1,15 &,- 04 &,- 74 &, 38 \\
\hline 3. Cuando me asignan lecturas, las leo la noche anterior. & 3,19 & 1,19 &,- 20 &,- 76 & 22 \\
\hline 4. Cuando me asignan lecturas, las reviso el mismo día de la clase. & 2,82 & 1,21 &, 10 &,- 79 &, 14 \\
\hline 5. Cuando tengo problemas para entender algo, inmediatamente trato de buscar ayuda & 2,32 & 1,19 & ,49 &,- 73 &, 45 \\
\hline 6. Asisto regularmente a clases. & 1,54 & 1,06 & 2,00 & 3,02 &, 17 \\
\hline 7. Trato de completar el trabajo asignado lo más pronto posible. & 2,05 & .99 &, 63 & -24 &, 53 \\
\hline 8. Postergo los trabajos de los cursos que no me gustan. & 2,36 & 1,21 &, 40 &,- 88 & 28 \\
\hline 9. Postergo las lecturas de los cursos que no me gustan & 2,22 & 1,19 &, 54 &,- 73 & ,32 \\
\hline 10. Constantemente intento mejorar mis hábitos de estudio & 1,96 & 1,06 &, 97 & 25 &, 50 \\
\hline 1 1. Invierto el tiempo necesario en estudiar aun cuando el tema sea aburrido. & 2,45 & 1,07 &, 26 & -62 &, 42 \\
\hline 12. Trato de motivarme para mantener mi ritmo de estudio. & 2,07 & 1,05 & 67 &,- 37 &, 58 \\
\hline 13. Trato de terminar mis trabajos importantes con tiempo de sobra. & 2,23 & 1,08 & 41 &,- 75 &, 54 \\
\hline 14. Me tomo el tiempo de revisar mis tareas antes de entregarlas. & 2,27 & 1,22 & ,63 &,- 58 &, 49 \\
\hline 15. Raramente dejo para mañana lo que puedo hacer hoy. & 2,93 & 1,23 &, 04 &,- 85 &, 00 \\
\hline $\begin{array}{l}\text { 16. Disfruto la mezcla de desafío con emoción de esperar hasta el último minuto } \\
\text { para completar una tarea. }\end{array}$ & 2,98 & 1,31 & ,02 & $-1,00$ &,- 02 \\
\hline
\end{tabular}

Nota: $\mathrm{DE}=$ desviación estándar; Asi.=asimetría; Curt.= curtosis; ritc=índice de correlación ítem-total corregido 
reactivos 4, 15 y 16, que obtuvieron pesos factoriales inferiores a .20 y se incluyeron las correlaciones entre los errores de medida de los ítems 8 y 9, que presentaron una modificación de índice de 226,51. En el segundo modelo re-especificado se retiraron los reactivos $1,3,4,6,8,9,15$ y 16 , que reportaron valores inferiores a .30. En el tercer modelo re-especificado se obtuvieron valores con mejor ajuste absoluto $\left(X^{2} / \mathrm{gl}=3\right.$; $\mathrm{GFI}=$,96; $\mathrm{RMSEA}=, 048$ y $\mathrm{SRMR}=0,34)$, un ajuste comparativo más favorable $(\mathrm{CFI}=, 98$ y $\mathrm{TLI}=$,97), además de un ajuste parsimonioso con valores inferiores al primer modelo $(\mathrm{AIC}=666,08$ y 68,98$)$.

En la Figura 1 se presentan los valores de pesos factoriales estandarizados del modelo M16-1, donde se reporta valores de .23 a .71 a excepción de los reactivos 4, 15 y 16 que presentan valores inferiores a 20 .

En la Figura 2 se muestran los valores del modelo M13-1, donde se reporta valores de .21 a .71, siendo el valor más bajo para el reactivo 7 y el más elevado para el reactivo 11, asimismo, el valor de los errores correlacionados (8 y 9) es de .78.

En la Figura 3 se muestran los pesos factoriales estandarizados del modelo M8-1, donde los valores van de .37 a .72.

\section{ANÁLISIS DE CONSISTENCIA INTERNA}

En la Tabla 3, se presenta los valores de consistencia interna según el coeficiente de consistencia interna Omega, de tal modo que el primero modelo presenta un valor de .73, el segundo modelo un valor de .77 y el tercer modelo de .80 , donde la valoración es

Tabla 2

Índices de ajuste global $(n=366)$

\begin{tabular}{|c|c|c|c|c|c|c|c|}
\hline \multirow{2}{*}{ Modelo } & \multicolumn{4}{|c|}{ Ajuste Absoluto } & \multicolumn{2}{|c|}{ Ajuste Comparativo } & \multirow{2}{*}{$\begin{array}{l}\text { Ajuste } \\
\text { Parsimonioso } \\
\text { AIC }\end{array}$} \\
\hline & $X^{2} / g \mid$ & GFI & RMSEA (IC 90\%) & SRMR & $\mathrm{CFI}$ & TLI & \\
\hline M16-1 & 5,79 & 85 & , $115(, 106-, 124)$ & ,093 & .61 & .55 & 666.08 \\
\hline M13-1 & 2,30 &, 94 & ,060 (,047-,072) & ,056 & ,93 & 91 & 201,13 \\
\hline M8-1 & 1,85 &, 96 &, $048(, 022-, 072)$ &, 034 &, 98 & 97 & 68,98 \\
\hline \multicolumn{8}{|c|}{$\begin{array}{l}\text { Nota: M16-1=Modelo de } 16 \text { ítems; M13-1=modelo de } 13 \text { ítems; M8-1=modelo de } 8 \text { ítems; X2/gl=Chi cuadrado sobre grados } \\
\text { libertad; GFI=índice de bondad de ajuste; RMSEA=error cuadrático medio de aproximación; SRMR=residuo cuadrático } \\
\text { estandarizado; CFI=índice de ajuste comparativo; TLI=índice de Tuker-Lewis; AIC=criterio de información de Akaike }\end{array}$} \\
\hline
\end{tabular}

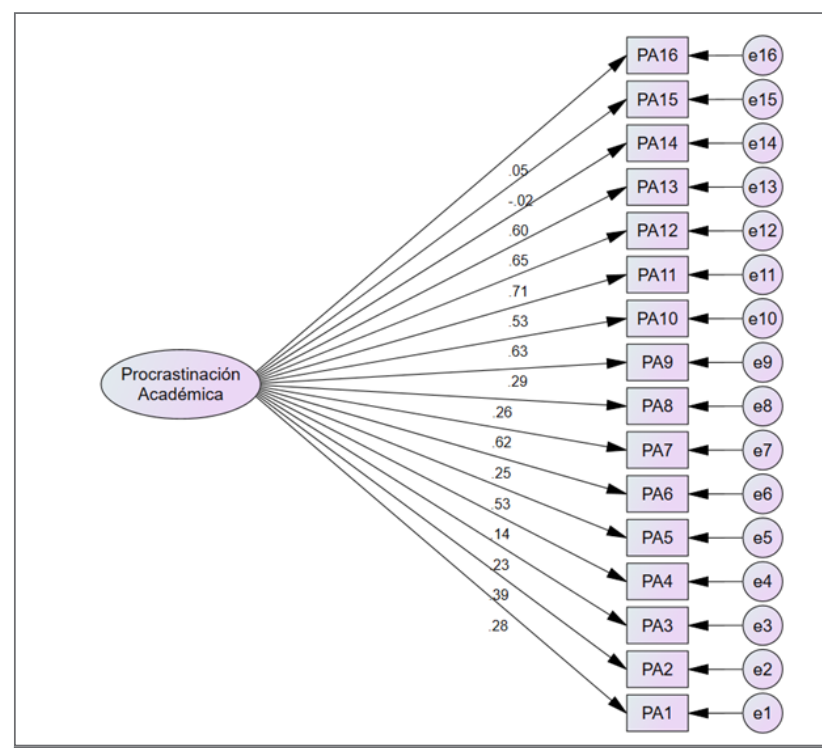

Figura 1: Estructura factorial del modelo M 16-1 ( N=366)

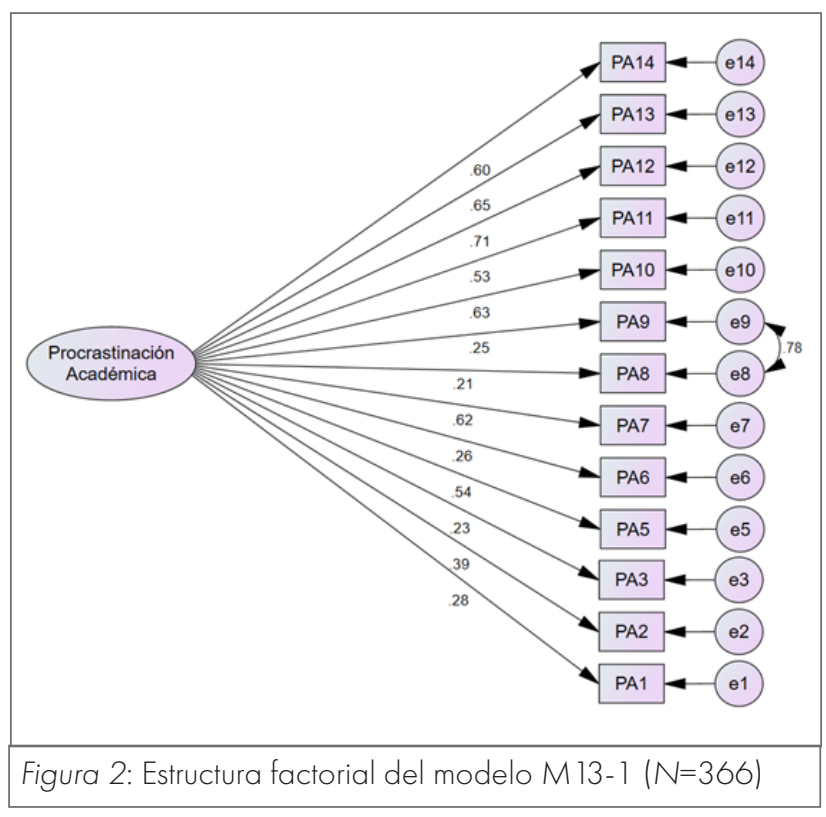


Tabla 3

Valores de consistencia interna $(\mathrm{N}=366)$

\begin{tabular}{|c|c|c|c|c|c|c|c|c|}
\hline Modelos & Nótem & $M$ & $D E$ & Asim. & Curt. & $\omega$ & \multicolumn{2}{|c|}{ IC 95\% } \\
\hline M16-1 & 16 & 39,12 & 8,05 &, 15 &, 01 &, 73 &, 69 &, 77 \\
\hline M13-1 & 13 & 30,39 & 7,58 &, 30 &,- 04 &, 77 &, 74 &, 81 \\
\hline M8-1 & 8 & 18,27 & 5,72 &, 46 &, 24 &, 80 &, 77 &, 84 \\
\hline
\end{tabular}

Nota: M16-1=Modelo de 16 ítems; M13-1=modelo de 13 ítems; M8-1=modelo de 8 ítems; $\omega=$ coeficiente de consistencia interna Omega; $M=$ media; $D E=$ desviación estándar; Asim.=asimetría; Curt.=curtosis; $I C=$ intervalos de confianza; LI=límite inferior; $\mathrm{LS}=$ límite superior

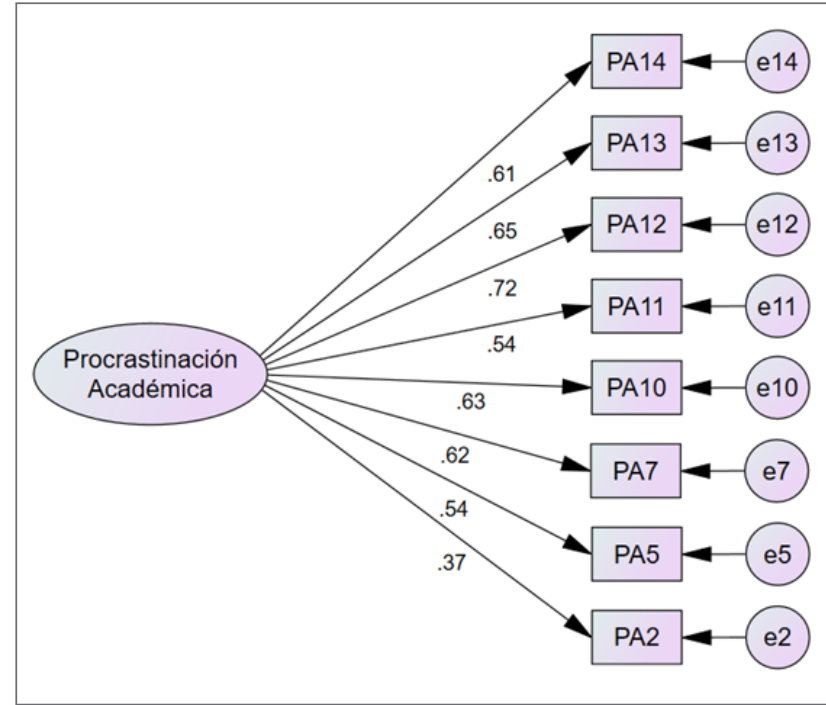

Figura 3: Estructura factorial del modelo M8-1 ( N=366) aceptable (Campo y Oviedo, 2008).

\section{DISCUSIÓN}

El propósito de este trabajo fue examinar las evidencias psicométricas de la Escala de Procrastinación Académica, estudiando la validez de constructo de la prueba mediante análisis factorial confirmatorio y la fiabilidad de la escala, en una muestra de estudiantes de Secundaria, y descartar la versión de 12 ítems de la Escala de Procrastinación Académica de Busko (1998), propuesta por Domínguez, Villegas y Centeno (2014) y en contraparte proponer una versión de 8 ítems.

Los resultados señalan que la Escala de Procrastinación Académica presenta índices de ajustes adecuados, a diferencia en otros estudios psicométricos realizados en muestra de universitarios y estudiantes de Secundaria (Domínguez, Villegas y Centeno 2014;
Guevara, 2017). Por otro lado, los resultados brindan evidencias satisfactorias de la estructura interna de la escala, confirmando su unidimensionalidad.

Respecto a la validez, se analizó la correlación ítem-test, hallando valores que varían de .22 a .58 (aceptables) en la EPA y por otro lado valores inferiores a ,20 (inaceptables) (Kline, 1993) en los reactivos $4,6,15$ y 16 . Similar resultado encontramos al de Domínguez, Villegas y Centeno (2014), que habían eliminado los reactivos $3,4,15$ y 16. A diferencia, Álvarez (2010) encuentra índices de correlación entre 0.31 y 0.54 (aceptable). Se muestra que en la asimetría, los ítems 6, 7, 10, 12 y 14 presentan valores superiores $(< \pm 1.5)$ y en la curtosis los ítems $2,3,4,5,6,8,11,13$ y 15 presentan valores superiores $(< \pm 1.5)$ reflejando ausencia de normalidad multivariada (Korkmaz, Goksuluk y Zararsiz, 2015). La eliminación de los ítems puede encerrar múltiples aspectos influyentes en la procrastinación académica en la muestra de estudiantes de secundaria y no se puede considerar concluyente debido a que el estudio ha evaluado colegios estatales y faltaría ver a las instituciones privadas. Además, hay que considerar que en el proceso de un estudio psicométrico se presentan eliminación de ítems de un instrumento (Muñiz, Elosua y Hambleton, 2013).

Por otro lado, los resultados del análisis factorial confirmatorio indican que los datos se ajustan a una dimensión de 8 ítems; Álvarez (2010) reporta que una dimensión de 16 times en concordancia con Busko (1998); a diferencia Domínguez, Villegas y Centeno 
(2014) reportan 2 dimensiones en el EPA, que los índices de bondad de ajuste eran mayores en un modelo bifactorial a comparación del propuesto por Álvarez (2010).

En base a los hallazgos, se evidencia que en la nueva versión EPA-8 se agrupan ítems vinculados a edad (estudiantes secundaria) a diferencia del grupo etario usado por Álvarez (2010) y Domínguez, Villegas y Centeno (2014) que aplicaron en una muestra universitaria. Se puede analizar que en los items 3 y 4 los sujetos del nivel de secundaria tienen una visión diferente a los universitarios. Debido a que los estudiantes de secundaria al presentar una falta a su clase encuentran mecanismos externos que le obligan a cumplir con sus tareas. En cambio en los universitarios sus mecanismos son libres y no obligatorio de cumplir en su mayoría conllevando a posterior a generar fracaso en su rendimiento asociado a una ansiedad por seguir postergando el cumplimiento de sus tareas. Al respecto, Chan (2011) estudio la Procrastinación Académica como predictor del rendimiento académico en una población de universitarios, que reporta una baja competencia académica, involucrado en no presentar los productos, y no estudiar para los exámenes. Solomon y Rothblum (1984) nos mencionaba que la procrastinación académica implica una situación de ansiedad debido a la postergación de las tareas.

Respecto a la fiabilidad por consistencia interna, se calculó el coeficiente Omega. Se observa en el tercer modelo re-especificado valores aceptables (Campo y Oviedo, 2008) para la escala en su versión unidimensional. Así mismo, se reportan similares índice de fiabilidad por Álvarez (2010) y Domínguez, Villegas y Centeno (2014), que usaron alfa de Cronbach. Los reportes en las diversas investigaciones nos indican que las puntuaciones obtenidas tiene una estabilidad en relación al instrumento que mide EPA.

El estudio presenta algunas limitaciones que las investigaciones futuras con la EPA deben tomar en consideración. En primer lugar, los sujetos del estudio no eran representativos de la totalidad de los estudiantes de Chimbote, debido que no se consideró a las instituciones educativas particulares. En segundo lugar, no se examinó otra propiedad en la fiabilidad como un test-retest, importante para evaluar la variabilidad de puntuaciones en el tiempo. Además, no se controló el sesgo de deseabilidad social que puede afectar la calidad de las respuestas.

Como conclusión, los hallazgos demuestran que el EPA (versión de 8 ítems) cuenta con evidencias psicométricas de validez y fiabilidad para ser considerada una medida de evaluación de la procrastinación académica para estudiantes de educación básica y recomendable para el trabajo en la práctica de la psicología educativa o en la investigación.

\section{- Conflicto de intereses.}

Los autores declaran no tener ningún conflicto de intereses.

\section{REFERENCIAS}

Álvarez, O. (2010). Procrastinación general y académica en una muestra de estudiantes de secundaria de Lima metropolitana. Revista Persona, 13, 159177.

Alegre, A. (2013). Autoeficacia y procrastinaciónacademica en estudiantes universitarios de Lima Metropolitana. Propósitos y Representaciones, 1(2), 57-82. http://dx.doi.org/10.20511/ pyr2013.v1n2.29

Ato, M., López-García, J. J., \& Benavente, A. (2013). Un sistema de clasificación de los diseños de investigación en psicología. Anales de Psicología, 29(3), 1038-1059. http://dx.doi.org/10.6018/ analesps.29.3.178511

Brown, T. A. (2006). Confirmatory factor analysis for applied research. Nueva York: The Guilford Press.

Busko, D. (1998). Causes and consequences of perfectionism and procrastination: A structural equation model. Tesis de maestría no publicada. Guelph, Ontario: University of Guelph.

Campo, A., \& Oviedo, H. (2008). Propiedades psicométricas de una Escala: La Consistencia Interna. Revista de Salud Pública, 10(5), 831 -839. 
Cardona, L. (2015). Relaciones entre procrastinación académica y estrés académico en estudiantes universitarios (Tesis de grado) Universidad de Antioquia, Medellín.

Carranza, R., \& Ramírez, A. (2013). Procrastinación y características demográficas asociados en estudiantes universitarios. Apuntes Universitarios, 3(2), 95- 108.

Chan, L. (201 1). Procrastinación académica como predictor e el rendimiento académico en jóvenes de educación superior. Revista temática psicológica, 7(1), 53-62.

Domínguez, S., Villegas, G., \& Centeno, S. (2014) Procrastinación académica: validación de una escala en una muestra de estudiantes de una universidad privada. Liberabit, 20(2), 293-304.

Durán, C., \& Moreta, C. (2017). Relación entre la procrastinación académica y la autorregulación emocional en una muestra de estudiantes universitarios de Psicología: Caso Pucesa. (Tesis de grado) Pontificia Universidad Católica de Ecuador.

Durand, C., \& Cucho, N. (2016). Procrastinación académica y ansiedad en estudiantes de una universidad privada de Lima Este (Tesis de grado) Universidad Peruana Unión.

Elosua, P. (2011). Prácticas de psicometría. Manual de procedimiento. Servicio Editorial de la Universidad del País Vasco.

Ellis, A., \& Knaus, W. J. (2002). Overcoming procrastination. NY: New American Library

Escobedo, M. T., Hernández, J. A., Estebané, V., \& Martínez, G. (2016). Modelos de ecuaciones estructurales: características, fases, construcción, aplicación y resultados. Ciencia \& Trabajo, 18(55), 16-22. $\quad$ http://dx.doi.org/10.4067/ S0718-24492016000100004

Ferrari, J., \& Emmons, R. (1995). Methods of procrastination and their relation to self-control and self-reinforcement: An exploratory study. Journal of Social Behavior and Personality, 10(1), 135.
Ferrando, J., \& Anguiano, C. (2010). El análisis factorial como técnica de investigación en Psicología. Papeles del psicólogo, 31 (1), 18-33.

Garzón, A., \& Gil, J. (2017). El papel de procrastinación académica como factor de la deserción universitaria. Revista Complutense de Educación, 28(1), 307 324.

http://dx.doi.org/10.5209/rev RCED.2017.v28.n1.49682

Guevara, P. (2017). Propiedades psicométricas de la escala procrastinación académica en adolescentes del distrito de Víctor Larco Herrera (Tesis de grado). Lima, Perú: Universidad César Vallejo.

Hair, J., Black, B., Babin, B, Anderson, R., \& Tatham, R. (2010). Multivariate Data Analysis 7th edition. New York: Pearson.

Hernández, G. (2016). Procrastinación académica, motivos de procrastinación y bienestar psicológico en alumnos de ingeniería industrial de una Universidad de Trujillo (Tesis de grado). Universidad Privada del Norte, Trujillo.

Hu, L., \& Bentler, P. (1998). Fit Indices in Covariance Structure Modeling: Sensitivity to Underparameterized Model Misspecification. Magazine Psychological Methods. 3(4), $424-453$.

Klassen, R., \& Kuzucu, E. (2009). Academic procrastination and motivation of adolescents in Turkey. Educational Psychology, 29(1), 69-81.

Kline, P. (1993). A Handbook of Test Construction. New York: Methuen.

Korkmaz, S., Goksuluk, D., \& Zararsiz, G. (2015). MVN: multivariate normality tests. $R$ packageversion, 4.

McDonald, R. (2013). Test theory: A unified treatment. New York: Psychology Press.

Muñiz, J., Elosua, P., \& Hambleton, R. (2013). Directrices para la traducción y adaptación de los tests: segunda edición. Psicothema, 25(2), 151-157.

https://doi: 10.7334 psicothema2013.24

Onwuegbuzie, A. (2004). Academic procrastination and statistics anxiety. Assessment \& Evaluation in Higher Education, 29(1), 3-19. 
Quant, D., \& Sánchez, A. (2012). Procrastinación, procrastinación académica: concepto e implicaciones. Revista Vanguardia Psicológica Clínica Teórica y Práctica, 3(1), 45-59.

Quinde,J.(2014).Propiedades psicométricas de la escala de procrastinación académica en universitarios entrerrianos. En VIII Congreso Internacional de Investigación y Práctica Profesional en Psicología XXIII Jornadas de Investigación de la Facultad de Psicología XII Encuentro de Investigadores (Vol. 6, No. 2, pp. 3568).

Rodriguez, A., \& Clariana, M. (2017). Procrastinación en estudiantes universitarios: su relación con la edad y el curso académico. Revista Colombiana de Psicología, 26(1), 45-60. https:// doi: 10.15446/rcp.v26n 1.53572

Ruiz, M. , Pardo, A., \& San Martín, R. (2010). Modelos de ecuaciones estructurales. Papeles del psicólogo, 37 (1), 34-45. http://dx.doi.org/10.4067/s071824492016000100004

Sánchez, A.(2010). Procrastinación académica: un problema en la vida universitaria. Revista studiositas, (5)2, 88-93.

Steel, P. (2007). The nature of procrastination: A meta-analytic and theoretical review of quintessential self-regulatory failure. Psychological Bulletin, 133(1), 65-94.
Steel, P., \& Ferrari, J. (2013). Sex, education and procrastination: an epidemiological study of procrastinators' characteristics from a global sample. European Journal of Personality, 27(1), 51-58.

Solomon, L. J., \& Rothblum, E. D. (1984). Academic procrastination: Frequency and cognitive-behavioral correlates. Journal of counseling psychology, 37 (4), 503.

https://doi.org/10.1037/00220167.31 .4 .503

Schouwenburg H. C. (2004). Perspectives on counseling the procrastinator. In H. C. Schouwenburg, C. H. Lay, T. A. Pychyl, \& J. R. Ferrari (Eds.), Counseling the procrastinator in academic settings (pp. 197-208). Washington, DC: American Psychological Association.

Tabachnick, B., Fidell, S., \& Ullman, J. (2007). Using multivariate statistics (Vol. 5). Boston, MA: Pearson.

Wheaton, B., Muthen, B., Alwin, D. \& Summers, G. (1977). Assessing reliability and stability in panel models. Sociological methodology, 8, 84-136.

http://dx.doi.org/10.2307/270754 